\title{
Cryopreparation of skin samples: A comparison between ultrathin cryo- sections and resin embedded sections of human skin
}

Roger Wepf, Stephan Pfeiffer*, Tobias Richter, Katja Dunckelmann, Stefan Biel, K.-P. Wittern; Beiersdorf AG, R\&D cosmed, Analytical Microscopy Dept., Unnastr. 48, 20245 Hamburg, *Institute of Botany, University of Kiel, Am Bot. Garten 5, 24098 Kiel, Germany

Preservation of skin in a "close-to -native state" without the loss of lipids can be achieved by high-pressure freezing, followed by cryo-sectioning and investigation in a Cryo-TEM (sections) or Cryo-SEM (remaining "bloc-face"). Therefore, vitrified samples were cryo sectioned at $100 \mathrm{~K}$ and investigated in the frozen-hydrated state at $103 \mathrm{~K}$. An alternative way to minimize loss of lipids and ultra structural changes, as determined by mass spectroscopy, is freeze-substitution in the presence of acetone saturated with uranyl acetate and embedding at low temperatures followed by sectioning and investigation at room temperature (1).

We compared the skin ultrastructure in cryosections (Fig. 3C $\sim 70 \mathrm{~nm}$ thickness) with that of skin in resin sections (Fig. 3A\&B) to determine the structural changes occurring with each procedure. We found ultrastructural details e.g. corneodesmosomes and lamellar bodies, in the process of fusion with the plasma membrane at the SC/S.granulosum interface in both types of sections. Cryosections show a strong deformation in the direction of the sectioning process. These deformations render study of the fine structure to a difficult task, and interpretation of fine details can only be performed in selected areas of a cryosection whereas no deformation of the structure could be found on the corresponding "bloc-face" observed by Cryo-SEM (2).

Our experience shows, nevertheless, that it is possible to investigate the ultrastructure of skin without the use of any chemical fixatives or dehydration techniques. But it also clearly reveals a significantly different lipid and protein (e.g. filaggrin) distribution when samples were chemically fixed prior to high pressure freezing (Fig.1\&2). In general lipids are redistributed over the entire epidermis during or after the chemical fixation process (Fig. 1A), whereas protein e.g. filaggrin are masked by the chemical fixative (Fig. 2A)!

Consequently, a routine comparison of the lipid structure and a better understanding of the ultrastructure of the viable skin and mainly the skin barrier are only possible by comparing EM and LM data (3) of skin biopsies, which have been prepared by "close-to-native state“ preserving preparation protocols and approved by cryosectioning for cryo-TEM.

\section{This work was supported by a German Government BMBF grant 13N7695/9}

\section{Literature:}

1) Pfeiffer S. et al. (2000) J. Invest Dermatol. 114, 1030-1038

2) T. Richter et al., Microscopy \& Microanalysis Proceedings 2003

3) S. Biel et al., Microscopy \& Microanalysis Proceedings 2003

Figure 1-3: High pressure frozen human skin biopsie; 1-3 A) chemical fixation prior to high pressure freezing followed by identical freeze substitution in Acetone/UrAc as for 1-3 B). $3 C$ Frozen hydrated TEM section. Labeling for filaggrin (1A-B) and glucosylceramid 3 (2AB). 


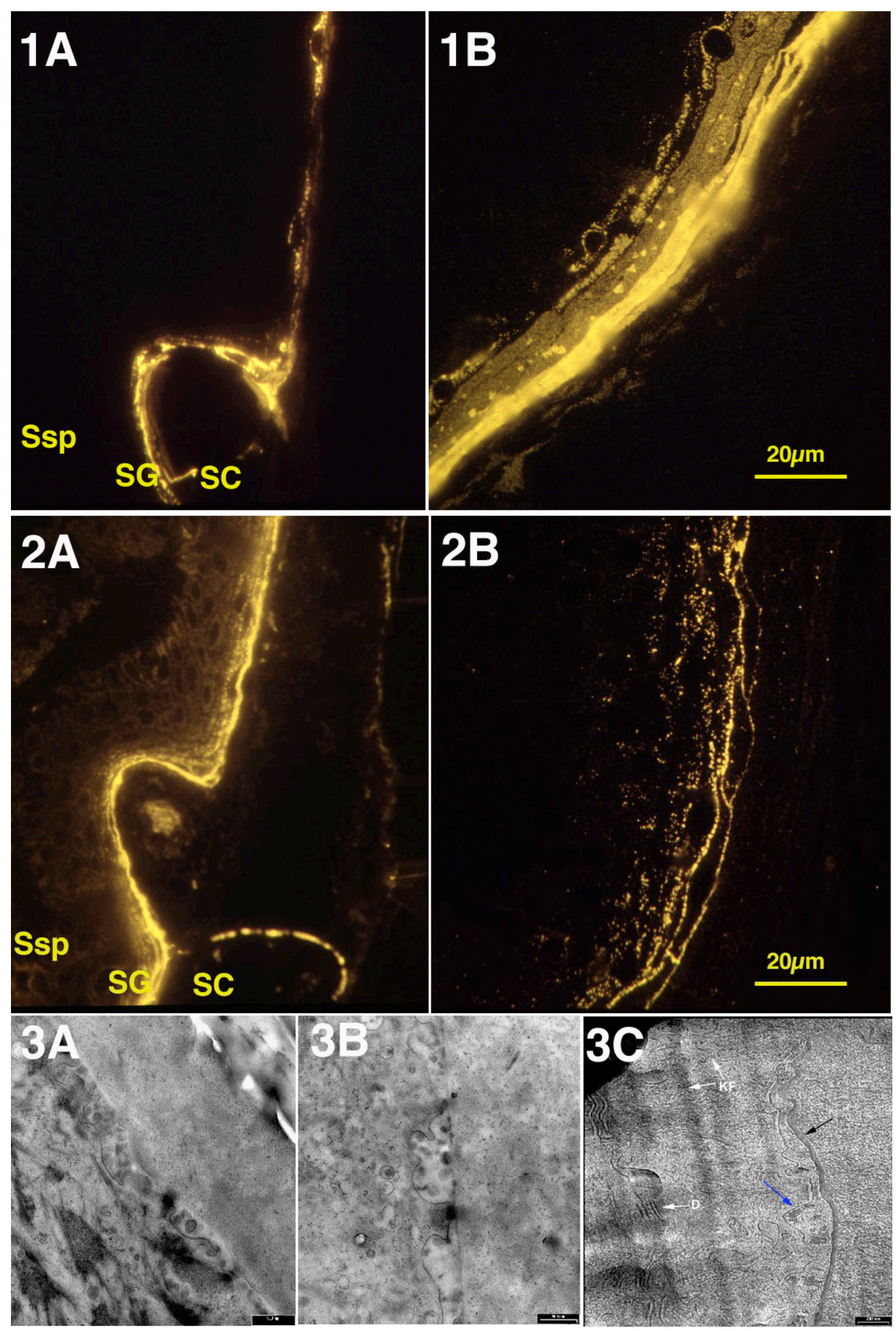

